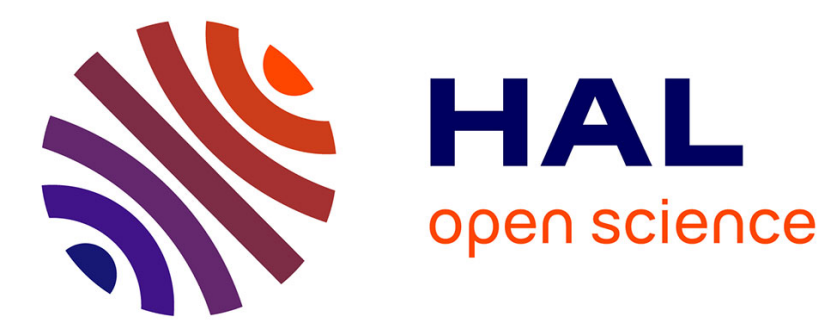

\title{
UWB Ranging for Rapid Movements
}

Tanguy Risset, Claire Goursaud, Xavier Brun, Kevin Marquet, Fabrice Meyer

\section{To cite this version:}

Tanguy Risset, Claire Goursaud, Xavier Brun, Kevin Marquet, Fabrice Meyer. UWB Ranging for Rapid Movements. IPIN 2018, Sep 2018, Nantes, France. pp.1-8, 10.1109/IPIN.2018.8533820 . hal01951454

\section{HAL Id: hal-01951454 \\ https://hal.inria.fr/hal-01951454}

Submitted on 14 Dec 2018

HAL is a multi-disciplinary open access archive for the deposit and dissemination of scientific research documents, whether they are published or not. The documents may come from teaching and research institutions in France or abroad, or from public or private research centers.
L'archive ouverte pluridisciplinaire HAL, est destinée au dépôt et à la diffusion de documents scientifiques de niveau recherche, publiés ou non, émanant des établissements d'enseignement et de recherche français ou étrangers, des laboratoires publics ou privés. 


\title{
UWB Ranging for Rapid Movements
}

\author{
Tanguy Risset*, Claire Goursaud*, Xavier Brun ${ }^{\dagger}$, Kevin Marquet * and Fabrice Meyer* \\ * Univ Lyon, INSA Lyon, Inria, CITI, F-69621 Villeurbanne, France \\ †Univ Lyon, INSA Lyon, AMPERE F-69621 Villeurbanne, France \\ Email: firstname.lastname@insa-lyon.fr
}

\begin{abstract}
Ultra Wide Band (UWB) provides ranging capabilities much more precise than other radio communication technologies. This paper presents experimental measurement of ranging precision between two UWB tags when one of the tag is moving fast. The use of a specific electro-pneumatic actuator provides precise ground truth for real distance. This study will allow to improve ranging for human wearable tags as for example sportsmen positionning or interaction between dancers during live performances. We show in particular how the inherent noisy measurement has to be smoothed to obtain more acurate ranging.
\end{abstract}

\section{INTRODUCTION}

Indoor wireless 3D positioning systems have recently gained a lot of attention. Many technologies have emerged and compete as the most precise, the cheapest or the least energy consuming one. They promise to provide new services in many different application domains such as surveying, ambient assistant living, drone navigation, health management, etc.

Requirements imposed to the positioning system may vary a lot depending on the intended application. We are interested in using precise indoor localization in industry [23] or entertainment. Arts and sports domains gained recently more attention as they provide new localization applications: precise localization of bikers in bike race races [16], dancers interacting with music and light based on their relative position, analysis and training of athletes movements [17], [21], etc. Many localization techniques rely on the basic distance measurements between two transceivers, so called ranging. The precision of the localization is highly impacted by the precision of the ranging. In this paper, we focus on the precision of UWB ranging rather than UWB localization.

Ultra Wide Band (UWB) is a wireless communication technology that is characterized, in its impulse radio scheme [25], by very short duration waveforms called pulses (in the order of few nanoseconds), using a wide band and low power spectral density. Among the many advantages offered by this technology is the fact that the arrival time of a pulse can be determined quite precisely, giving the opportunity to measure the distance between two communicating devices by estimating the flight time of the signal.

Although this technology has been known for a long time, it is only recently that cheap UWB chips have been commercialized for civilian applications (see the companies mentioned in Section II). As the UWB technology is sensitive to many parameters, the effective performance of localization systems based on UWB may vary a lot compared to what is announced in datasheets. Some accuracy studies have been performed [13], [14] but few of them focus on rapid movement of the transceivers.

Indeed, indoor ranging is in itself dependent on many parameter and very difficult to evaluate objectively, but when the transceivers are moving fast (say as if they were attached to dancer's wrists), more parameters are to be taken into account: transceiver calibration, random errors, presence of obstacle, antenna orientation etc.

In this paper, we study experimentally the precision of UWB ranging for rapid movements in an indoor environment, based on the technology proposed by Decawave (DW1000 [6]) whose chips have already been integrated in many commercial devices. We show in particular how to improve the precision of the distance measured by averaging the ranging over successive samples.

The paper is organized as follows: state of the art of indoor localization is given in Section II, UWB and UWB ranging is presented in Section III. Our experimental setup using a specific machine for fast movement measurements is presented in Section IV. The results of the experiments are presented in Section $\mathrm{V}$ before concluding remarks.

\section{STATE OF THE ART}

UWB has been foreseen for a long time as a key technology for ranging and 3D positioning. In 2001, Gezici and Poor wrote a survey on position estimation with UWB [8]. Since that work, lots of research papers and surveys have been published. In [10], Gu et. al. provided a survey on many techniques for indoor positioning. In [18], a classification of systems and localization schemes (triangulation, scene analysis, etc.) for wireless indoor positioning systems was proposed. Two recent surveys provide an exhaustive view of indoor positioning technologies [3] and UWB-based positioning technologies [1]. These studies basically agree on the fact that indoor localization is still an open problem, and this is partly because there are so many different available technologies that can be used.

Comparing two positioning systems is a complex task because it involves very different technical solutions - WLAN, UWB, sound, magnetic, vision, infrared etc. Moreover, many metrics have been proposed to evaluate a positioning system: accuracy, precision, power consumption, cost, form factor etc. Another parameter of great importance for localization is the 
position reconstruction algorithm from raw data (distance measurement, SINR, image etc.). The most frequent approaches for indoor localization are either vision-based solutions requiring complex cameras setup, or beacon-based solutions where a set of wireless sensors are placed at fixed locations and one sensor is the object to locate (Local Positioning Systems LPS) [19].

Approaches using ultrasound or UWB radio signals are reputed to be the most accurate beacon-based LPS localization solutions. Ultrasound is limited in range (about 10 meters) and cannot penetrate walls. UWB positioning systems have a larger coverage (more than $100 \mathrm{~m}$ ) but, as shown here and in various studies, UWB ranging is very sensible to obstacles. Outliers in non line-of-sight (NLOS) can be larger than one meter [12].

Among the first companies to propose UWB products are Time Domain or Ubisense. More recently, Bespoon and Decawave proposed cheap integrated solutions for UWB ranging. Decawave (DW1000) and BeSpoon (Spoonphone) use the IEEE 802.15.4a impulse radio standard as physical layer, while Ubisense (series 7000 IP sensors) and Time Domain (FIFE UWB chip) use their own custom-made communication and localization solutions.

After the release of these commercial solutions, several practical studies have been published, testing commercial chips in realistic environments to stress their performances in term of ranging and localization [13], [9], [2], [11], [26], [21]. In [21], experiments focussing on indoor positionning for sports postures are carried out using Pozyx Labs commercial board inluding a Decawave DW1000 chip. The authors study distributed algorithms for position reconstruction (Kalman or particuler filter). This work also shows that position accuracy estimation is difficult to evaluate because one needs a ground truth position value which is hard to obtain in fast movements. In our work, we have used a specific electro-pneumatic actuator, described in Section IV-B that provides millimeter-level exact positionning.

In [13], Hammer et. al. provide an important experimental study comparing static and dynamic positioning performances of two UWB systems (Decawave DW1000 and Time Domain P410) and one audio-based system. Their conclusion was that the Decawave system was well-suited for dynamic application and they also emphasize its low power consumption compared with the Time Domain system. Another study [14] compares Decawave DW1000 and BeSpoon solutions. The systems were declared roughly equivalent despite the fact that the size of antennas were different.

One important challenge today concerns the non-line-ofsight (NLOS) environment. In previous studies as well as in [28], it is mentioned that localization or even ranging with UWB in NLOS is "still a challenging problem for indoor locations". Complex methods using machine learning for detecting NLOS packet receptions are studied in [22]. A simpler and less precise method used in [21], [20], [27], [11] makes use of the power difference between the received power level and the power for the first path signal, these two power levels can be computed from metrics provided by the
Decawave chip [6].

So far, the only precise experience with moving UWB tags is the work of Hammer et. al [13], but their study targets localization which might be influenced by many sources of noise. Our study attempts to give a precise statistical model of UWB ranging precision during rapid movements. We also show that a good knowledge of Decawave firmware is mandatory for precise calibration and finally we provide insights on how to handle the inherent noise present in the distance measurements between two UWB tags.

\section{UWB AND RANGING}

Since the initial works of the American Army in the eighties, the UWB technology has been progressively regulated, and is now standardized in the IEEE 802.15.4 standard [24] that breaks down under two branches: I-UWB and MC-UWB. The first one, finally retained in IEEE standard, consists in sending Gaussian pulses of very short duration, thus using a large frequency band, in the order of the gigahertz.

The recommended modulation technique is a combination of a BPM (burst position modulation) and a BPSK (Binary Phase-Shift Keying) making feasible very simple transmitters with low cost and energy consumption. The frequency band is divided into 15 channels of variable sizes: the so-called lowfrequency channels (from $3.1 \mathrm{GHz}$ to $4.8 \mathrm{GHz}$ ), the so-called high-frequency channels (from $6.0 \mathrm{GHz}$ to $10.6 \mathrm{GHz}$ ) and the sub-gigahertz channel Frequency band from $249.6 \mathrm{MHz}$ to $749.6 \mathrm{MHz}$ ). Each of them must support the following speeds: $110 \mathrm{kbps}, 851 \mathrm{kbps}, 6.81 \mathrm{Mbps}$ and 27.24 Mbps [24].

Several methods allow to measure the distance between two UWB tags (Time Of Arrival, Time of flight measurements, Time Difference of Arrival, Angle of Arrival...). They all use the fact that a very precise timestamp can be associated with each pulse emission or reception and each of them tries to minimize the error coming from the fact that synchronization between two tags is not perfect. The method referenced in IEEE standard is the Symmetric Double Sided Two Way Ranging (SDS-TWR) which requires three messages to assert a distance between the tags [15].

Given that SDS-TWR is available on most commercial UWB tags, there remain several parameters that can be adjusted when designing a ranging dedicated to a specific positioning system. The most important is ranging measurement speed (we refer to it as ranging sampling rate). We were able to execute up to 283 ranging measurements per second with Decawave DW100 (283 Hz sampling rate), but this is useful only if the tag is moving fast. Other parameters are: the UWB mode (i.e. channel used and throughput), the antenna quality and orientation, and some calibration parameters such as the antenna delay that has to be very precisely set in order to minimize the error.

Our goal in this paper is to provide further insights following Hammer et. al. work [13] concerning the possible ranging precision obtained with moving UWB tags. In particular we show that it is worth smoothing the distance measured over several samples, because of the random noise present in 


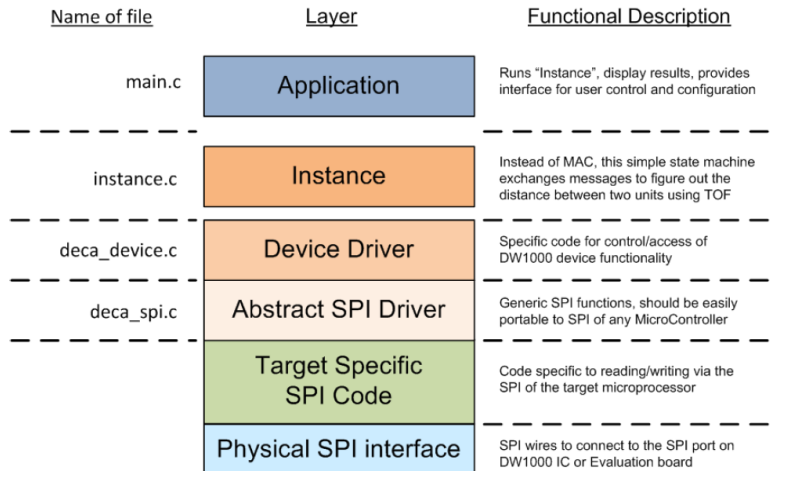

Fig. 1. Software layer for programming Decawave EVK1000, from [5].

measurements. We show that a very precise trajectory can be followed (with a few centimeters as mean error, and small standard deviation), even at $2 \mathrm{~m} . \mathrm{s}^{-1}$ speed.

\section{EXPERIMENTAL SETUP}

\section{A. Decawave DW1000}

The UWB platform used is the Decawave DW1000 integrated into the evaluation kit EVK1000 [7]. The DW1000 [6] is a low power radio chip that permits Standard UWB IEEE802.15.4-2011 communications. The DW1000 consists of an analog front-end radio with a transmitter and a receiver and a digital back-end which communicates with the external micro-controller running the application. The EVK1000 board is composed of a micro-controller ARM STM32F105RBT6, a LCD screen, and a DW1000 chip connected on the SPI peripheral of the ARM CPU. In addition, the kit comes with an open-source code called Decaranging for performing SDSTWR with various features that can be configured using the jumpers of the board. Those features include running the Anchor (i.e. master) code or running the Remote tag (i.e. mobile tag) code.

Having access to the whole source code, from the applicative code down to the low-level library for communicating between the STM32 CPU and the DW1000 chip, allowed us to understand the performance results. Fig. 1 shows the software stack provided by Decawave; users have access to the code of any layer with $\mathrm{C}$ files mentioned. The Decaranging software can be adapted to Linux using the gcc-arm-none-eabi compiler. Some compilation flags have to be tuned and some files have to be added to Decawave code: the CMIS files (Cortex Micro-controller Software Interface Standard) mandatory to STM32 programming and the startup file startup_stm32f10x_cl.s. The EVK1000 kit has been programmed using the STLINK JTAG device from ST-microelectronics and the stlink software from Texane.

A serial communication between the tag and the PC can be established when the tag is powered from the PC by the USB cable. We have used this serial port to recover in real time the distance measured by the Anchor.
We have modified the Decaranging code in the following way: suppress some LCD printing on the tag, control the sampling rate of measurement by setting the value of the POLL_SLEEP_DELAY constant to 0 (we were able to obtain a measuring sampling rate of $283 \mathrm{~Hz}$, much more than what is usually announced in similar studies). We were not able to have the predefined mode "fast onboard ranging" running with our system, hence we had to disable it (Switch S12). Most of the measurements were performed in mode 4 (switches S1-4 and S1-5 on): channel 2 with a 6.3 Mbps Data Rate [6], [7]. Finally the antenna delay was tuned by changing the DWT_PRF_64M_RFDLY constant to value 515.080f.

\section{B. Electro-pneumatic actuator}

In order to estimate the localization accuracy of a moving device, we need a controlled trajectory. We used an electropneumatic actuator built in the Ampere Laboratory [4], see Fig. 2 and Fig. 3. It combines two servo-valves used to precisely control the gas mass flow rates into a pneumatic cylinder which moves a carriage in linear displacement. The piston generates an arbitraty trajectory along one direction, constrained to an amplitude up to $50 \mathrm{~cm}$. This trajectory can be programmed in advance in term of position, velocity and acceleration variations. In addition, the effective piston position is monitored by the electro-pneumatic actuator. The data that we compared with UWB distance measurement is the effective piston position (i.e. real position), and not the predicted position as there might be small differences because of inertia.
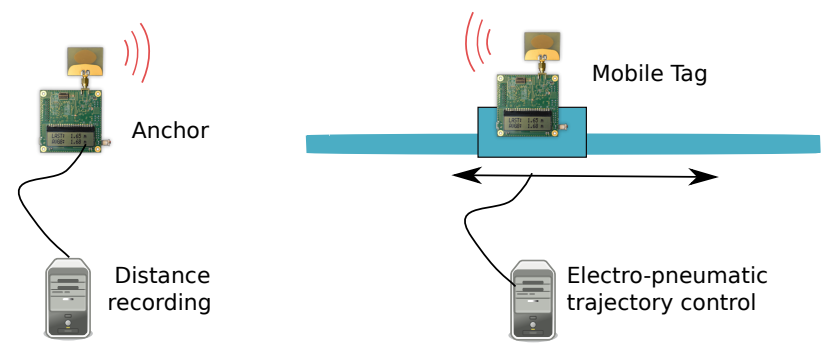

Fig. 2. Experimental setup for evaluating Decawave UWB ranging using the electro-pneumatic actuator [4]

\section{Experimental setup}

The results of Section V-B (dynamic ranging) were performed with the setup presented in Fig. 2. The distance measured by the UWB tags between the anchor and the mobile tag is recorded on the PC during 30 seconds approximately, for various speeds and distances. It is compared afterwards with the real distance reconstructed from the information logged by the electro-pneumatic piston. A picture of the experimental setup is shown on Fig. 3.

\section{Experimental plan}

Two types of measurements were performed. First a static experience was launched with the two tags being at a fixed distance (from one meter to twenty meters), in order to assert 


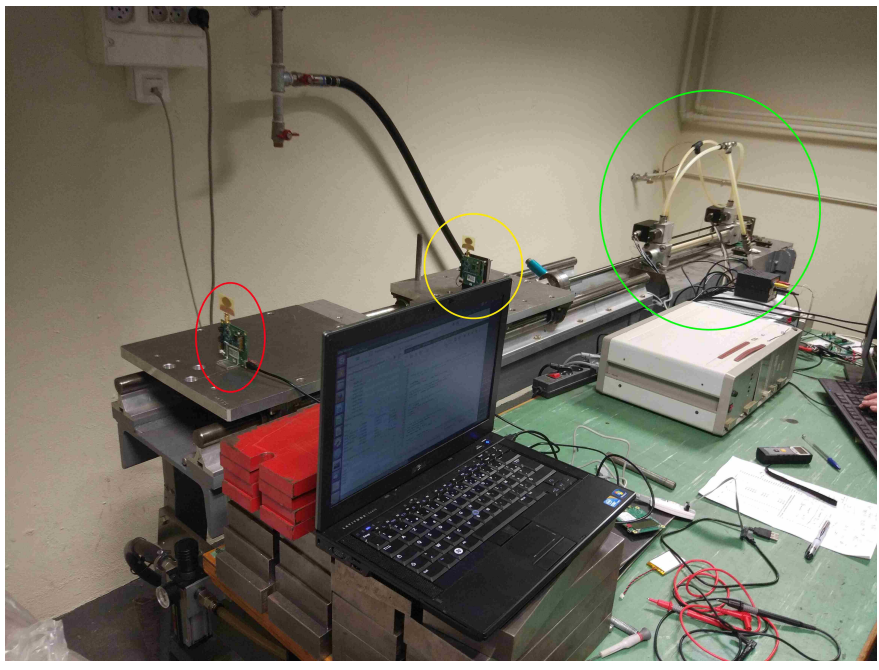

Fig. 3. Photo of the experimental setup, the two Decawave tags are surrounded as well as the pneumatic piston.

that the calibration of the tags was correct. Then all the other measurements were dynamic: one tag moving as shown on Fig. 3, the distance between the two tags oscillating around the central value of $92 \mathrm{~cm}$.

The piston was programmed to realize a sinusoidal movement at two speeds. The high speed, with an amplitude maximum displacement of the tag with respect to the center position - of $20 \mathrm{~cm}$, gave a maximal speed of approximately $2 \mathrm{~m} . \mathrm{s}^{-1}$ corresponding to a rapid human arm movement. The low speed was at $0.5 \mathrm{~Hz}$ with an amplitude of $30 \mathrm{~cm}$ corresponding approximately to a $0.5 \mathrm{~m} . \mathrm{s}^{-1}$ speed. For each experience, the measurement was realized twice at low speed and twice at high speed.

The measurements were done for various sampling rates (remind that we call sampling rate the rate at which the distance is measured): from 5 samples per second up to 285 samples per second. Some measurements were done with an obstacle and some with the two antennas not being properly aligned. The UWB data throughput used was set either at $6.8 \mathrm{Mb} / \mathrm{s}$ (mode 4 ) or at $110 \mathrm{~Kb} / \mathrm{s}$ (mode 3 ). As it implied to change a jumper on the board, we only made one measurement at $110 \mathrm{~Kb} / \mathrm{s}$, at a $83 \mathrm{~Hz}$ sampling rate.

\section{EXPERIMENTAL RESULTS}

\section{A. Static Ranging}

The results of the static ranging experiments are presented on Fig. 4. At each distance, the raw distance measured were averaged over 500 samples. The accuracy of the measurement (i.e. error with respect to the real distance) is below $1.5 \%$ of the distance, which is very precise. The precision of the measurements (i.e. variance of the error), is also very good: below $0.2 \%$ of the distance measured. This experiment was repeated four times, with various conditions (people moving around the tags but keeping line of sight clear), leading to similar results. These measurements provide us the static average ranging accuracy that can be obtained with our UWB
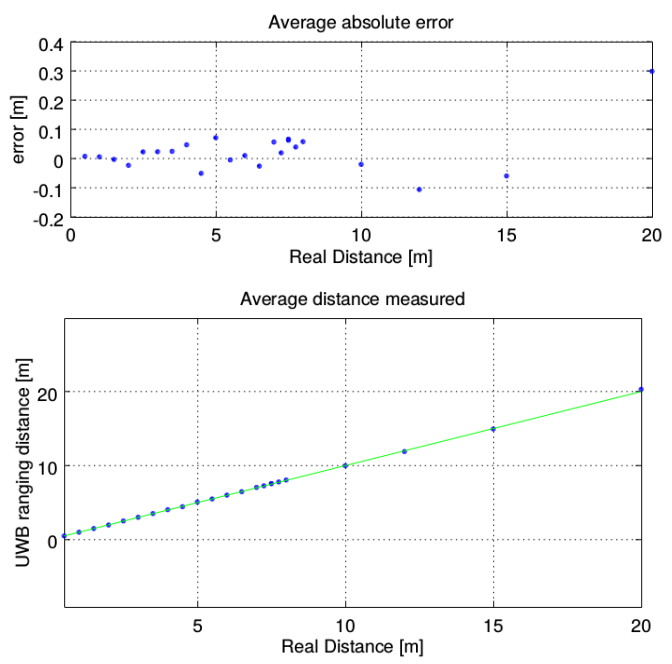

Fig. 4. UWB ranging distance measured in static scenario (mean over 500 samples). The error is always less than $1.5 \%$ of the distance and error variance always less than $0.2 \%$ of the distance.

tags: below $2 \%$ of the distance (i.e. $1,5 \mathrm{~cm}$ at $1 \mathrm{~m}$, or $30 \mathrm{~cm}$ at $20 \mathrm{~m}$ ), which is twice better than the accuracy announced in [21]. This accuracy difference is either linked to the fine tuning of decaranging firmware code and in particular the calibration of antenna delay (all our tags were calibrated with the same value of DWT_PRF_64M_RFDLY: 515.080f), or inherent to the different hardware used (Trek1000 Decawave board in our study, Pozyx labs development board in [21]).

\section{B. Dynamic Ranging Data processing}

For the second type of experiments, with a moving tag as sketched on Fig. 2, two streams of distance were obtained, one from the piston actuator and one from the UWB anchor tag. These two streams are represented on Fig. 5. It can be seen on Fig. 5 that some data preprocessing is necessary before comparing these two curves. Data processing concern three inconsistencies between the two curves: the sampling rate, the distance shift and the temporal shift.

- Sampling rate: the samples obtained from the two sources do not have the same sampling instants, the piston curve sampling rate is much higher. In order to obtain two curves with the same sampling instants, we have resampled the piston curve using the sampling date of the anchor tag. Each new sample created is deduced from the two surrounding samples by a linear interpolation. As the piston sampling rate was quite high, the introduced error is negligible.

- Distance shift: the data from the piston is first inverted and aligned to the data of the tag. It corresponds to the average distance 0.92 on the right of Fig. 5 (tag-anchor distance at when moving tag is idle) instead of 0 on the left of Fig. 5 (piston position when moving tag is idle).

- Temporal shift: as the clock of the PC and the electropneumatic machine are not synchronized (i.e. two dates 

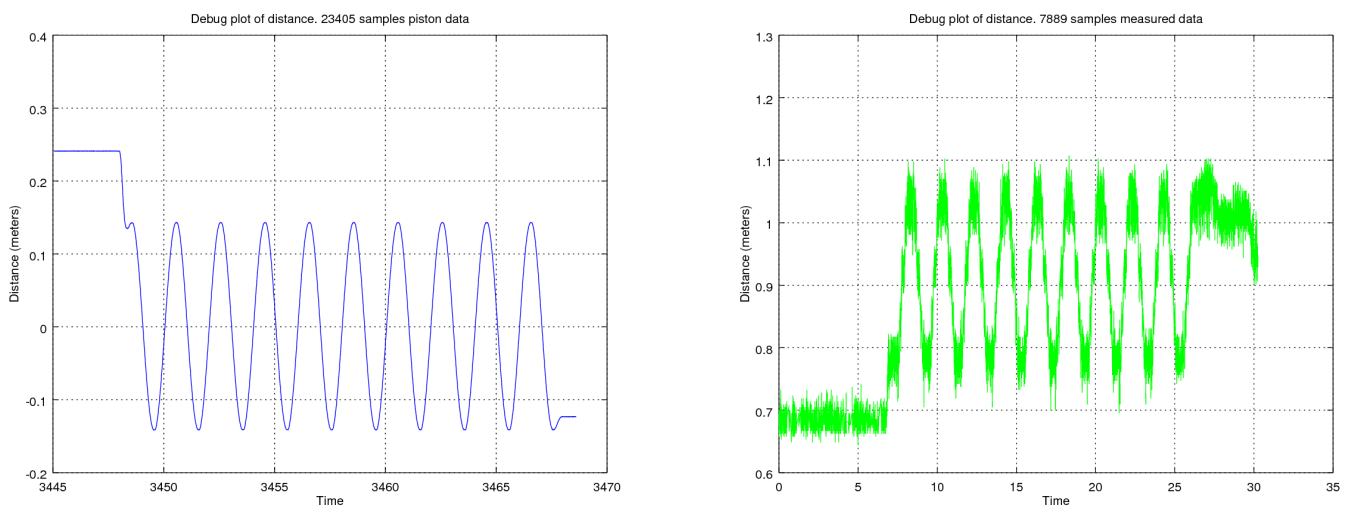

Fig. 5. Raw data obtained, from the piston (left) and from the UWB ranging (right), at slow speed with $283 \mathrm{~Hz}$ sampling rate. The two curves have to be aligned in time (X-axis) and space (Y-axis) and aligned on the same sampling rate.

in abscissa of the two curves of Fig. 5 do not correspond to the same physical instant), we align in time the two curves. To do that, we apply several cross-correlations between the two signals and deduce the temporal shift that minimizes the error between the two curves. The result of this alignment can be seen on Fig. 6: the curves from the piston and from the measurements are superposed. Fortunately, the clock drift between the two systems was not significant enough during the duration of the each measurements (30s). It might be necessary to take it into account for longer experiments.

If we are to do ranging in real-time, a little delay occurs between the distance measurement and its arrival on the PC (through a serial link). Unlike previous work [13], we did evaluate this delay and did not integrate it into the ranging error because we believe that this is very application-dependent. However we have taken into account the delay induced by the averaging over several samples proposed in Section V-D.

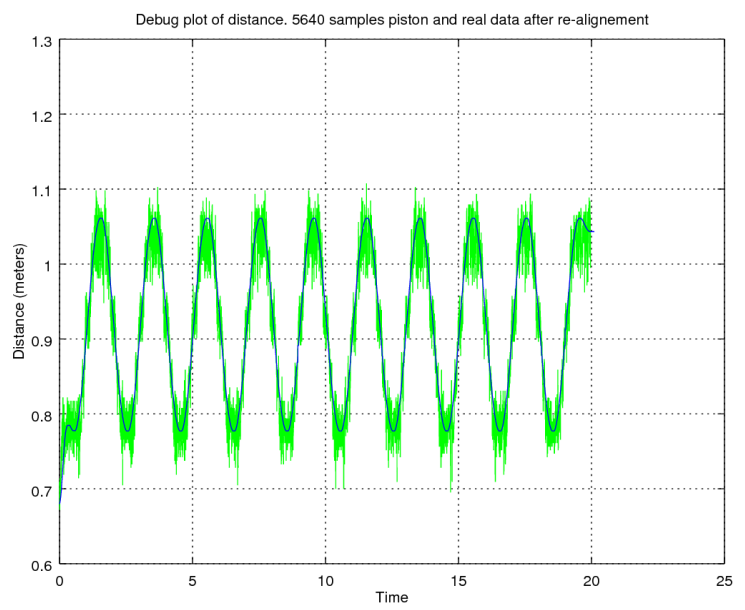

Fig. 6. The two data streams superimposed: raw data in green, real distance in blue (slow speed, $283 \mathrm{~Hz}$ sampling rate).

\section{Dynamic Ranging Results}

As mentioned in Section IV, several parameters have been explored: the sampling rate, the tag speed (fast or slow), the antenna orientation, and the presence of obstacles. The results are presented in Table I to Table IV. We recall that each measurement was done twice, the number reported here being the average between the two measurements (no significant difference was observed between two similar experiments). The data obtained at $83 \mathrm{~Hz}$ sampling rate was produced tested with a UWB different bit rate $(110 \mathrm{~kb} / \mathrm{s})$, all other data are obtained with $6,3 \mathrm{Mb} / \mathrm{s}$ UWB bit rate.

Table I reports the distance error in dynamic UWB ranging - fast displacement speed - for various sampling rates. One can notice that the maximum error can be quite large $(13 \mathrm{~cm}$ when the tags are at a distance between $70 \mathrm{~cm}$ and $110 \mathrm{~cm}$ ), but the mean error is quite low (between 2 and $4 \mathrm{~cm}$ ) and gets better when the sampling rate is smaller, except at $5 \mathrm{~Hz}$, but this is due to the fact that the sampling is too close to the Shannon sampling bound - the piston having a $2 \mathrm{~Hz}$ sinusoidal - , and the temporal shift pre-processing described above is not precise enough in that case. This experiment highlights the fact that high sampling rate could be used to provide offline trajectory reconstruction because the mean error is quite low.

\begin{tabular}{|c|c|c|c|}
\hline Sampling Rate & Max Error & Mean Error & Std dev. \\
\hline $283 \mathrm{~Hz}$ & $13.35 \mathrm{~cm}$ & $3.01 \mathrm{~cm}$ & $2.40 \mathrm{~cm}$ \\
$143 \mathrm{~Hz}$ & $12.51 \mathrm{~cm}$ & $2.96 \mathrm{~cm}$ & $2.41 \mathrm{~cm}$ \\
$83 \mathrm{~Hz}(110 \mathrm{~kb} / \mathrm{s})$ & $15.1 \mathrm{~cm}$ & $2.54 \mathrm{~cm}$ & $1.92 \mathrm{~cm}$ \\
$48 \mathrm{~Hz}$ & $8.8 \mathrm{~cm}$ & $2.35 \mathrm{~cm}$ & $1.65 \mathrm{~cm}$ \\
$5 \mathrm{~Hz}$ & $9.8 \mathrm{~cm}$ & $3.6 \mathrm{~cm}$ & $2.35 \mathrm{~cm}$ \\
\hline
\end{tabular}

STATISTIC OF DYNAMIC RANGING IN FAST MOVEMENT SCENARIO (2 $\left.m . s^{-1}\right), 92 \mathrm{CM}$ DISTANCE AND $20 \mathrm{CM}$ MOVEMENT AMPLITUDE

Table II presents the same measurements with a slower speed of the moving tag: $0.5 \mathrm{~m} . \mathrm{s}^{-1}$. As expected, the average error is a little bit better $(2.21 \mathrm{~cm}$ instead of $3.01 \mathrm{~cm}$ at $283 \mathrm{~Hz}$ sampling rate), and the standard deviation is a little bit better too. However, we are in the same accuracy category: a relative error between $2 \%$ and $4 \%$, which is better than 
what is announced in the technical documentation and in many paper [13], [14], [21].

\begin{tabular}{|c|c|c|c|}
\hline Sampling Rate & Max Error & Mean Error & Std dev. \\
\hline $283 \mathrm{~Hz}$ & $10.25 \mathrm{~cm}$ & $2.21 \mathrm{~cm}$ & $1.71 \mathrm{~cm}$ \\
$143 \mathrm{~Hz}$ & $10.11 \mathrm{~cm}$ & $2.22 \mathrm{~cm}$ & $1.67 \mathrm{~cm}$ \\
$83 \mathrm{~Hz}(110 \mathrm{~kb} / \mathrm{s})$ & $13.15 \mathrm{~cm}$ & $2.48 \mathrm{~cm}$ & $1.87 \mathrm{~cm}$ \\
$48 \mathrm{~Hz}$ & $8.4 \mathrm{~cm}$ & $2.2 \mathrm{~cm}$ & $1.6 \mathrm{~cm}$ \\
$5 \mathrm{~Hz}$ & $8.7 \mathrm{~cm}$ & $3.3 \mathrm{~cm}$ & $2.1 \mathrm{~cm}$ \\
\hline
\end{tabular}

STATISTIC OF DYNAMIC RANGING IN SLOW MOVEMENT SCENARIO $\left(0.5\right.$ m.s $\left.{ }^{-1}\right), 92$ CM DISTANCE AND 30 CM AMPLITUDE

Table III shows the influence of an obstacle. As mentioned previously in the paper and confirmed in other studies, a real obstacle (human or metallic) greatly deteriorates ranging performances because of radio multipath in indoor ranging. A real-time positioning algorithm should integrate a strategy to exclude outliers concerning only one tag as they probably correspond to temporary obstacles.

\begin{tabular}{|c|c|c|c|}
\hline Sampling rate & Max error & Mean error & Std dev. \\
\hline None & $10.25 \mathrm{~cm}$ & $2.21 \mathrm{~cm}$ & $1.71 \mathrm{~cm}$ \\
Cardboard & $10.75 \mathrm{~cm}$ & $2.45 \mathrm{~cm}$ & $2.2 \mathrm{~cm}$ \\
Human & $75.65 \mathrm{~cm}$ & $8.45 \mathrm{~cm}$ & $9.05 \mathrm{~cm}$ \\
\hline
\end{tabular}

TABLE III

OBSTACLE INFLUENCE AT SLOW SPEED, WITH $283 \mathrm{HZ}$ SAMPLING FREQUENCY

Table IV shows the influence of the antenna orientation which is, as for tag speed, not very important. Of course, this is very dependent on the antenna design, the ones used in Decawave kit being quite large.

\begin{tabular}{|c|c|c|c|}
\hline Antenna Orient. & Max error & Mean error & Std dev. \\
\hline $0^{\circ}$ & $10.25 \mathrm{~cm}$ & $2.21 \mathrm{~cm}$ & $1.71 \mathrm{~cm}$ \\
$45^{\circ}$ & $13.8 \mathrm{~cm}$ & $3 \mathrm{~cm}$ & $2.25 \mathrm{~cm}$ \\
$92^{\circ}$ & $14.4 \mathrm{~cm}$ & $3.25 \mathrm{~cm}$ & $2.5 \mathrm{~cm}$ \\
\hline
\end{tabular}

INFLUENCE OF THE ANTENNA ORIENTATION. THE FIRST COLUMN REPRESENTS THE ANGLE BETWEEN THE ANTENNAS OF THE ANCHOR AND THE MOVING TAG. SLOW SPEED, 283 Hz SAMPLING RATE

\section{Filtering Noise}

As can be seen on the right of Fig. 5, the UWB ranging raw data is impacted by an error that behaves like noise. As far as we noticed, this is always the case in UWB ranging measurements: a noise exist, that looks random. An example of the distribution of the measured distance error is shown in Fig. 7, for the measurement done at slow speed with $283 \mathrm{~Hz}$ sampling rate (distance between the tags oscillating around 92 $\mathrm{cm})$.

This inherent noise implies that two successive measurements can be quite different, and as we will show, averaging (or smoothing) the values measured over some successive samples improve the accuracy of the distance obtained.

Fig. 6 shows the superposition of the raw data from the piston and the anchor. Fig. 8 is drawn from the same data but with a smoothing of anchor measurement data, i.e. data was averaged over 40 samples. It seems obvious from these two

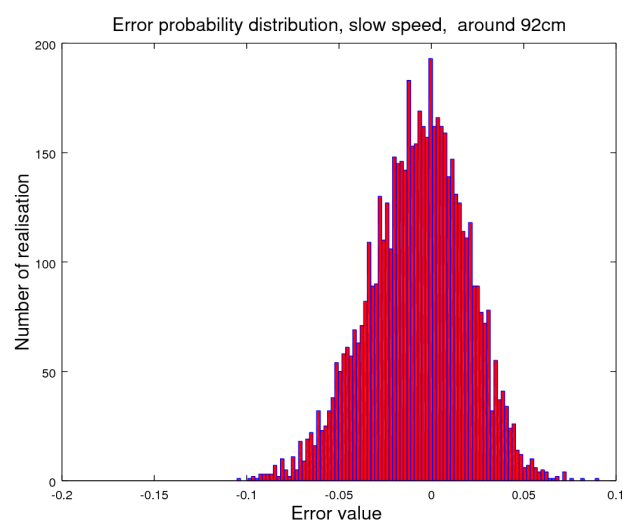

Fig. 7. Error distribution on a particular experiment (slow speed, $283 \mathrm{~Hz}$ sampling rate, distance between the tags oscillating around $92 \mathrm{~cm}$ ).

figures that averaging will help a lot in a process of trajectory reconstruction for instance. We provide hereafter a study about the impact of different averaging on accuracy of the measured distance.

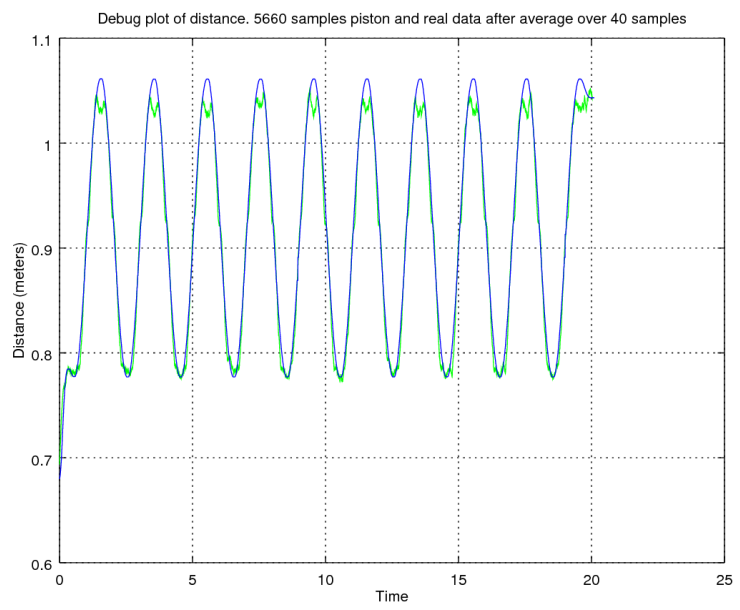

Fig. 8. Superposition of real distance (blue) and smoothed measured distance averaged over 40 samples (slow speed, $283 \mathrm{~Hz}$ sampling rate), this has to be compared with raw data shown on Fig. 6

\begin{tabular}{|c|c|c|c|}
\hline Sampling Feq. & max Error & Mean Error & Std Dev. \\
\hline $283 \mathrm{~Hz}$ & $4.02 \mathrm{~cm}$ & $1.06 \mathrm{~cm}$ & $0.89 \mathrm{~cm}$ \\
$143 \mathrm{~Hz}$ & $3.63 \mathrm{~cm}$ & $1.23 \mathrm{~cm}$ & $0.81 \mathrm{~cm}$ \\
$83 \mathrm{~Hz}($ mode $110 \mathrm{kB} / \mathrm{s})$ & $6.55 \mathrm{~cm}$ & $2.15 \mathrm{~cm}$ & $1.05 \mathrm{~cm}$ \\
$48 \mathrm{~Hz}$ & $5.2 \mathrm{~cm}$ & $1.1 \mathrm{~cm}$ & $0.7 \mathrm{~cm}$ \\
\hline
\end{tabular}

INFLUENCE OF THE SMOOTHING OVER 40 SAMPLES FOR A SLOW SPEED MOVING TAG, THE OBTAINED ACCURACY HAS TO BE COMPARED WITH TABLE II, ALMOST TWICE BETTER.

The results obtained by smoothing on the slow speed scenario is shown in Table $\mathrm{V}$ and should be compared with Table II. These results show that the accuracy of the measurements can be improved by $50 \%$ by averaging over successive measurements. For instance smoothing measurements over 40 
samples (at $283 \mathrm{~Hz}$ sampling) improves the average error from $2.21 \mathrm{~cm}$ to $1.06 \mathrm{~cm}$, an improvement of more than $50 \%$. The standard deviation and maximum error are improved as well. This is one major contribution of the paper: averaging successive distance samples can improve a lot the accuracy of the ranging with UWB technology.

However, the number of successive samples over which averaging should depend on the measurement frequency and on the speed of the device: for instance, at $5 \mathrm{~Hz}$ it does not make sense to average over 40 samples - i.e. over 8 seconds - except if the tag is almost static. Moreover, the results of Table V have been obtained offline, i.e. the two curves have been aligned offline as it was done on Fig. 8. This is possible if the ranging is computed after all samples have been obtained. If, on the contrary, the ranging has to be used online (e.g. in a live performance of dancers for instance), averaging adds a delay corresponding to the time needed to wait for the needed samples to compute the average.
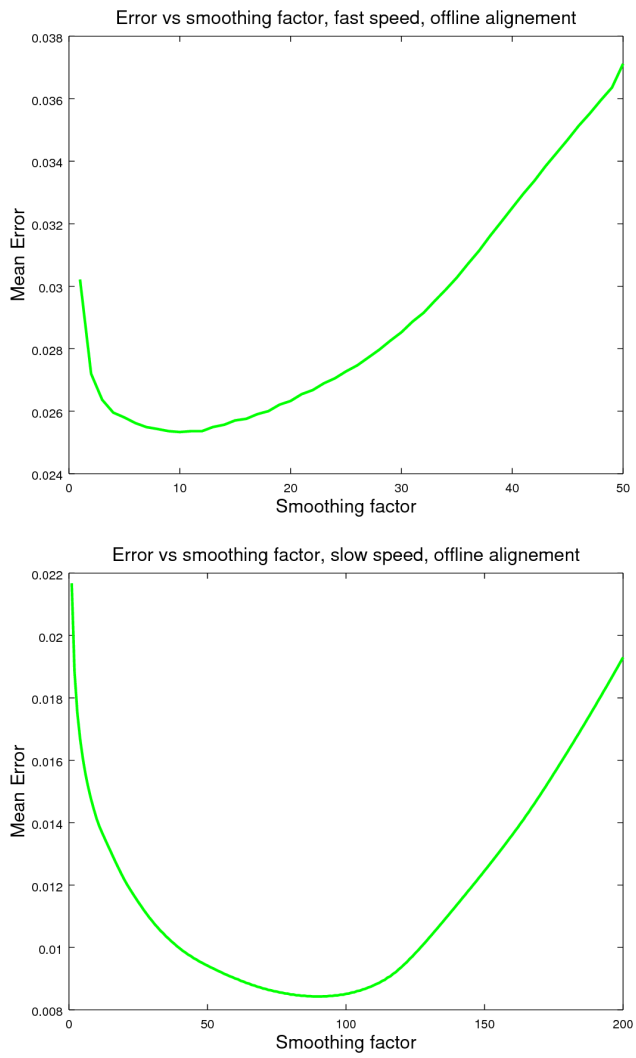

Fig. 9. Effect of the smoothing factor on the mean error of the measured distance, in fast and slow scenario at $283 \mathrm{~Hz}$ sampling, when smoothing is performed offline.

Fig. 9 shows distance error mean for various smoothing factors for offline ranging in fast and slow scenarios at $283 \mathrm{~Hz}$ sampling. The accuracy is optimal when the mean is computed over 10 samples at $2 m . s^{-1}$ and over 85 samples at $0.5 m . s^{-1}$. Fig. 10 identifies the optimal smoothing factor in the same conditions but in the online ranging, where averaging implies waiting for more samples while the tag is still moving. In the online case, the smoothing still improves the accuracy (5 samples at $2 \mathrm{~m} . \mathrm{s}^{-1}$, and 10 samples at $0.5 \mathrm{~m} . \mathrm{s}^{-1}$ ).

These experiments show that the accuracy of UWB ranging is dependent on many parameters. Very precise accuracy and precision can be obtained but highly depending on the conditions: speed of the tags and sampling rate in particular (and line of sight of course). A complete system that performs online ranging should associate an accelerometer to tune these parameters in real time in order to obtain accurate ranging results with a dynamic adaptation of the sampling rate. A high sampling rate improves the quality of the trajectory estimation but is also highly energy consuming, hence a dynamic trade-off should be decided online with the help of the accelerometer. Future work should propose a coupling between accelerometer and ranging sampling rate to optimize accuracy and power consumption of UWB ranging.
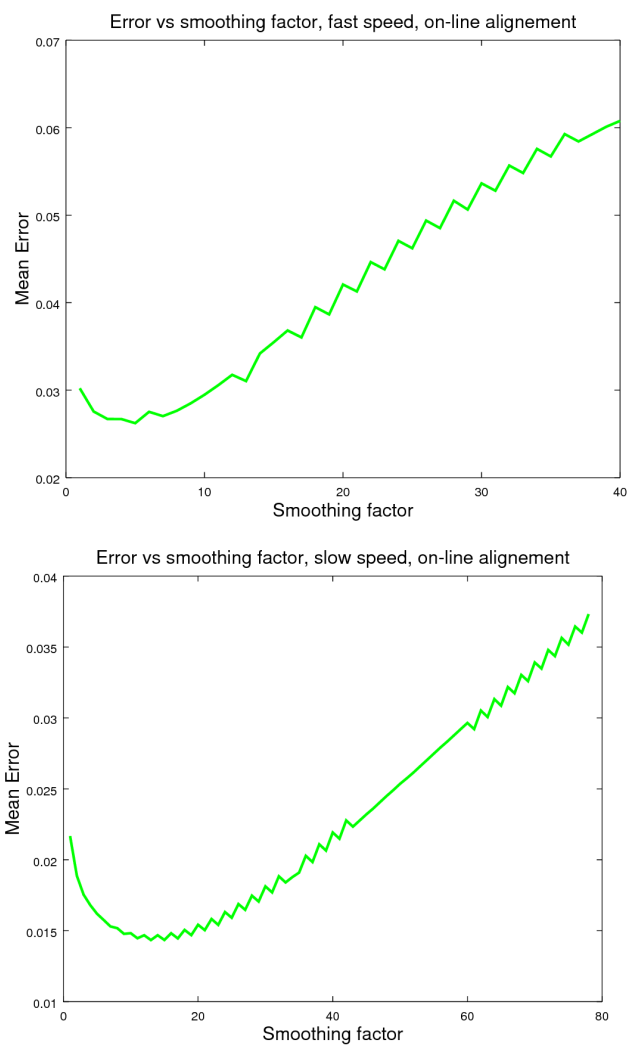

Fig. 10. Effect of different values of smoothing factor on the mean error of the measured distance, in fast and slow scenarios, when smoothing is performed online. The stair case shape is due to the alternations of odd and even smoothing factor

\section{CONClusion}

We have performed a precise study of UWB moving tags ranging accuracy and precision, mostly oriented towards application related to physical activities (sports, arts).

This study confirms some results that were previously obtained: the orientation of the antenna does not have a huge impact on precision of ranging in UWB, whereas obstacles 
have a huge impact on precision. It shows that a very good accuracy (approximately $1 \mathrm{~cm}$ ) can be obtained with careful UWB parameter tuning and it demonstrates an important new experimental result: the precision of the measurements can be improved by $50 \%$ by averaging over successive (fast) measurements.

\begin{tabular}{|c|c|c|c|}
\hline $\begin{array}{c}\text { mean distance } \\
\text { error }\end{array}$ & Static & $\begin{array}{c}\text { Slow } \\
\left(\simeq 0.5 m . s^{-1}\right)\end{array}$ & $\begin{array}{c}\text { Fast } \\
\left(\simeq 2 m . s^{-1}\right)\end{array}$ \\
\hline without smoothing & $1.5 \mathrm{~cm}$ & $2.21 \mathrm{~cm}$ & $3.01 \mathrm{~cm}$ \\
\hline online, optimal smoothing & - & $1.44 \mathrm{~cm}$ & $2.62 \mathrm{~cm}$ \\
\hline offline, optimal smoothing & - & $0.83 \mathrm{~cm}$ & $2.53 \mathrm{~cm}$ \\
\hline
\end{tabular}

SUMMARY OF THE BEST AVERAGED ERROR OBTAINED IN VARIOUS CONDITION FOR TAGS DISTANT OF APPROXIMATELY 92CM (AT $283 \mathrm{~Hz}$ SAMPLING RATE).

A brief summary of the ranging performances obtained at approximately $92 \mathrm{~cm}$ distance between the tags is recalled in Table VI. This table exemplifies the main contribution of the paper: we show how the inherent noise of UWB distance measurement can be reduced and how the accuracy of ranging can be improved by smoothing. We obtained here up to $65 \%$ accuracy improvement in slow speed, offline alignment for instance.

A natural consequence of this study will be to associate an accelerometer to UWB ranging devices, not only to tune measurement sampling rate, but also measurement smoothing parameter.

\section{REFERENCES}

[1] A. Alarifi, A. Al-Salman, M. Alsaleh, A. Alnafessah, S. Al-Hadhrami, M. A. Al-Ammar, and H. S. Al-Khalifa. Ultra wideband indoor positioning technologies: Analysis and recent advances. Sensors, 16(5), 2016.

[2] K. Batstone, M. Oskarsson, and K. Åström. Towards real-time time-ofarrival self-calibration using ultra-wideband anchors. In 2017 International Conference on Indoor Positioning and Indoor Navigation (IPIN), Sept 2017.

[3] R. F. Brena, J. P. García-Vázquez, C. E. Galván-Tejada, D. MuñozRodriguez, C. Vargas-Rosales, , and J. James Fangmeyer. Evolution of indoor positioning technologies: A survey. Journal of Sensors, (2630413), 2017.

[4] X. Brun and D. Thomasset. Choice of control law in electropneumatics. expertise using an industrial benchmark and some new trends. In Proceedings of the 39th IEEE Conference on Decision and Control (Cat. No.00CH37187), volume 2, pages 1323-1328 vol.2, 2000.

[5] Decawave. Decaranging arm source code guide, 2014.

[6] Decawave. Dw1000 datasheet, 2014 V2.04.

[7] Decawave. Evk1000 user manual, 2015 1.10.

[8] S. Gezici and H. V. Poor. Position estimation via ultra-wide-band signals. Proceedings of the IEEE, 97(2):386-403, Feb 2009.

[9] V. Gharat, E. Colin, G. Baudoin, and D. Richard. Indoor performance analysis of lf-rfid based positioning system: Comparison with UHFRFID and UWB. In 2017 International Conference on Indoor Positioning and Indoor Navigation (IPIN), Sept 2017.

[10] Y. Gu, A. Lo, and I. Niemegeers. A survey of indoor positioning systems for wireless personal networks. IEEE Communications Surveys Tutorials, 11(1):13-32, First 2009.

[11] K. Gururaj, A. K. Rajendra, Y. Song, C. L. Law, and G. Cai. Realtime identification of NLOS range measurements for enhanced UWB localization. In 2017 International Conference on Indoor Positioning and Indoor Navigation (IPIN), Sept 2017.

[12] İ. Güvenç, C.-C. Chong, F. Watanabe, and H. Inamura. NLOS identification and weighted least-squares localization for UWB systems using multipath channel statistics. EURASIP Journal on Advances in Signal Processing, 2008(1), Aug 2007.
[13] F. Hammer, R. Yudanto, K. Neumann, M. Pichler, J. Cockx, C. Niestroj, and F. Petré. Performance evaluation of 3d-position estimation systems. IEEE Sensors Journal, 16(16):6416-6424, Aug 2016.

[14] A. R. Jiménez and F. Seco. Comparing decawave and bespoon UWB location systems: Indoor/outdoor performance analysis. In 2016 International Conference on Indoor Positioning and Indoor Navigation (IPIN), pages 1-8, Oct 2016.

[15] M. Kwak and J. Chong. A new double two-way ranging algorithm for ranging system. In 2010 2nd IEEE InternationalConference on Network Infrastructure and Digital Content, pages 470-473, Sept 2010.

[16] M. Lauzier, A. Fraboulet, J.-M. Gorce, and T. Risset. Live group detection for mobile wireless sensor networks. In Ninth International Conference on Body Area Networks, London, Great Britain, Sept. 2014.

[17] R. Leser, A. Baca, and G. Ogris. Local positioning systems in (game) sports. Sensors, 11(10):9778-9797, 2011.

[18] H. Liu, H. Darabi, P. Banerjee, and J. Liu. Survey of wireless indoor positioning techniques and systems. IEEE Transactions on Systems, Man, and Cybernetics, Part C (Applications and Reviews), 37(6):10671080, Nov 2007.

[19] R. Mautz. Indoor positioning technologies. PhD thesis, 2012.

[20] A. K. Rajendra, Y. Song, K. Gururaj, C. X. Wang, G. F. Cai, C. L. Law, W. P. Tay, and H. P. Chow. UWB based real-time cooperative localization system. In 2017 International Conference on Indoor Positioning and Indoor Navigation (IPIN), Sept 2017.

[21] M. Ridolfi, S. Vandermeeren, J. Defraye, H. Steendam, J. Gerlo, D. D. Clercq, J. Hoebeke, and E. D. Poorter. Experimental evaluation of UWB indoor positioning for sport postures. Sensors, 18(1):168, 2018.

[22] V. Savic, E. G. Larsson, J. Ferrer-Coll, and P. Stenumgaard. Kernel methods for accurate UWB-based ranging with reduced complexity. IEEE Transactions on Wireless Communications, 15(3):1783-1793, 2016.

[23] B. Silva, Z. Pang, J. Åkerberg, J. Neander, and G. Hancke. Experimental study of UWB-based high precision localization for industrial applications. In 2014 IEEE International Conference on Ultra-WideBand (ICUWB), pages 280-285, Sept 2014.

[24] I. C. Society. Ieee standard for low-rate wireless networks. IEEE Std 802.15.4-2015, pages 446-474, 2015.

[25] M. Z. Win and R. A. Scholtz. Impulse radio: how it works. IEEE Communications Letters, 2:36-38, 1998.

[26] Y. Yang, C. Zhang, P. Dai, X. Bao, L. Han, and Q. Wang. Analysis and discrimination of ranging error based on obstacle experiments. In 2017 International Conference on Indoor Positioning and Indoor Navigation (IPIN), Sept 2017.

[27] Y. Yang, C. Zhang, P. Dai, X. Bao, L. Han, and Q. Wang. Analysis and discrimination of ranging error based on obstacle experiments. In 2017 International Conference on Indoor Positioning and Indoor Navigation (IPIN), Sept 2017.

[28] T. Ye, M. Walsh, P. Haigh, J. Barton, A. Mathewson, and B. O'Flynn. An experimental evaluation of ieee 802.15.4a ultra wide band technology for precision indoor ranging. Int. J. Ambient Comput. Intell., 4(2):48-63, Apr. 2012. 